\title{
On the Solution of Local Fractional Differential Equations Using Local Fractional Laplace Variational Iteration Method
}

\author{
Pranay Goswami ${ }^{1}$ and Rubayyi T. Alqahtani ${ }^{2}$ \\ ${ }^{1}$ School of Liberal Studies, Ambedkar University Delhi, Delhi 110006, India \\ ${ }^{2}$ Department of Mathematics and Statistics, College of Science, Al-Imam Mohammad Ibn Saud Islamic University (IMSIU), \\ Riyadh 11566, Saudi Arabia \\ Correspondence should be addressed to Pranay Goswami; pranaygoswami83@gmail.com
}

Received 24 August 2015; Revised 19 November 2015; Accepted 24 December 2015

Academic Editor: Laura Gardini

Copyright (C) 2016 P. Goswami and R. T. Alqahtani. This is an open access article distributed under the Creative Commons Attribution License, which permits unrestricted use, distribution, and reproduction in any medium, provided the original work is properly cited.

We present iteration formulae of a fractional space-time telegraph equation using the combination of fractional variational iteration method and local fractional Laplace transform.

\section{Introduction}

Fractional differential equations have number of properties and play master role in different fields of study. These equations and their solutions gained remarkable interest due to its striking role in every science and technology [14]. Various fields like diffusion, transport theory, scattering theory, rheology, quantitative biology, and so forth are successfully applying fractional differential equations to define and explain number of phenomena but fractional calculus is not perfectly applicable in the case of fractal functions. In order to deal with fractal problems in various fields, the concept of local fractional derivative was developed. The local fractional calculus was introduced by Yang and further applications of this derivative can be found in the references [5-8]. There are variety of analytical methods for solving them like Adomian decomposition method, generalized differential transform method, homotopy perturbation method, separating variables, local fractional Laplace method, local fractional Sumudu method, variational iteration method, and fractional variational iteration method [5, 6, 9-14].

In this paper, we develop an iteration formula to solve generalized fractional space-time telegraph equation by using the combination of fractional variational iteration method and local fractional Laplace transform.

\section{Definitions and Mathematical Preliminaries}

Definition 1 (see $[7,8]$ ). The partition of interval $[a, b]$ is denoted as $\left(t_{j}, t_{j+1}\right), j=0, \ldots, N-1, t_{0}=a$, and $t_{N}=b$ with $\Delta t_{j}=t_{j+1}-t_{j}$ and $\Delta t=\max \left\{\Delta t_{0}, \Delta t_{1}, \ldots\right\}$. Local fractional integral of $f(x)$ in the interval $[a, b]$ is given by $[7,8]$ :

$$
\begin{aligned}
{ }_{a} I_{b}^{(\alpha)} f(x) & =\frac{1}{\Gamma(1+\alpha)} \int_{a}^{b} f(t)(d t)^{\alpha} \\
& =\frac{1}{\Gamma(1+\alpha)} \lim _{\Delta t \rightarrow 0} \sum_{j=0}^{N-1} f\left(t_{j}\right)\left(\Delta t_{j}\right)_{\alpha} .
\end{aligned}
$$

Definition 2. The local fractional derivative of $f(x) \in$ $C_{\alpha}(a, b)$ of order $\alpha$ at $x=x_{0}$ is defined [see $\left.[7,8]\right]$ as

$$
\begin{aligned}
f^{(\alpha)}\left(x_{0}\right) & =\left.\frac{d^{\alpha}}{d x^{\alpha}} f(x)\right|_{x=x_{0}} \\
& =\lim _{x \rightarrow x_{0}} \frac{\Delta^{\alpha}\left(f(x)-f\left(x_{0}\right)\right)}{\left(x-x_{0}\right)^{\alpha}},
\end{aligned}
$$

where $\Delta^{\alpha}\left(f(x)-f\left(x_{0}\right)\right) \cong \Gamma(\alpha+1) f(x)-f\left(x_{0}\right)$. 
Definition 3. The local fractional Laplace transform of $f(x)$ of order $\alpha$ is defined [15] as

$$
\bar{L}_{\alpha}[f(x)]=\frac{1}{\Gamma(1+\alpha)} \int_{0}^{\infty} E_{\alpha}\left(-s^{\alpha} x^{\alpha}\right) f(x)(d x)^{\alpha},
$$

$$
0<\alpha \leq 1
$$

where $x \in C$ and $E_{\alpha}(x)$ is Mittage Leffler function $\sum_{k=0}^{\infty}\left(x^{k} / a k !\right)$.

And inverse local fractional Laplace transform is defined as

$$
\bar{L}_{\alpha}^{-1}\{f(s)\}=f(x), \quad 0<\alpha \leq 1 .
$$

On using the above definition of local fractional Laplace transform, the following result can easily be obtained [15]:

$$
\begin{aligned}
\bar{L}_{\alpha}\left\{f^{n \alpha}(x)\right\} & =s^{n \alpha} \bar{L}_{\alpha}\{f(x)\}-\sum_{k=1}^{n} s^{(k-1) \alpha} f^{(n-k) \alpha}(0), \\
\bar{L}_{\alpha}\left\{x^{k \alpha}\right\} & =\frac{\Gamma(1+k \alpha)}{s^{(k+1) \alpha}} .
\end{aligned}
$$

\section{The Local Fractional Laplace Variational Iteration Method}

This section introduces the idea of local fractional Laplace variational method for the following fractional space-time telegraph equation:

$$
D_{0+}^{n \alpha} v(x, t)=L_{\alpha}(v(x, t))+N_{\alpha}(v(x, t))+f(x, t),
$$

where $0<\alpha \leq 1, x, t \geq 0, L_{\alpha}$ is a linear operator, $N_{\alpha}$ is nonlinear operator, and $f$ is a source term.

Taking local fractional Laplace transform of (6), both sides with respect to " $x$ " are as follows:

$$
\begin{aligned}
& s^{n \alpha} \bar{L}_{\alpha}\{f(x)\}-\sum_{k=1}^{n} s^{(k-1) \alpha} f^{(n-k) \alpha}(0) \\
& \quad=\bar{L}_{\alpha}\left[L_{\alpha}(v(x, t))+N_{\alpha}(v(x, t))+f(x, t)\right] .
\end{aligned}
$$

For an algebraic equation, the iteration formula can be constructed as

$$
x_{n+1}=x_{n}+\lambda^{\alpha} f\left(x_{n}\right) .
$$

The optimality condition for the extreme $\delta x_{n+1} / \delta x_{n}=0$, leads to

$$
\lambda^{\alpha}=-\frac{1}{f^{\prime}\left(x_{n}\right)},
$$

where $\delta$ is the classical variational operator.
By the formula of (8), we get the iteration formula for (7) as follows:

$$
\begin{aligned}
& \bar{L}_{\alpha} v_{n+1}(s, t)=\bar{L}_{\alpha} v_{n}(s, t)+\lambda^{\alpha}\left\{s^{n \alpha} \bar{L}_{\alpha}\left\{v_{n}(s, t)\right\}\right. \\
& -\sum_{k=1}^{n} s^{(k-1) \alpha} v_{n}^{(n-k) \alpha}(0, t) \\
& \left.\quad-\bar{L}_{\alpha}\left[L_{\alpha}\left(v_{n}(x, t)\right)+N_{\alpha}\left(v_{n}(x, t)\right)+f(x, t)\right]\right\} .
\end{aligned}
$$

Put $\lambda^{\alpha}=-s^{-n \alpha}$, the Lagrange multiplier [16] in (10):

$$
\begin{gathered}
\bar{L}_{\alpha} v_{n+1}(s, t)=\left\{\left[\sum_{k=1}^{n} s^{(k-1-n) \alpha} v_{n}^{(n-k) \alpha}(0, t)\right]\right. \\
+s^{-n \alpha} \bar{L}_{\alpha}\left[L_{\alpha}\left(v_{n}(x, t)\right)+N_{\alpha}\left(v_{n}(x, t)\right)\right. \\
+f(x, t)]\} .
\end{gathered}
$$

Taking inverse local fractional Laplace transform into account, we arrived at

$$
\begin{aligned}
& v_{n+1}(x, t)=\bar{L}_{\alpha}^{-1}\left\{\left[\sum_{k=1}^{n} s^{(k-1-n) \alpha} v_{n}^{(n-k) \alpha}(0, t)\right]\right. \\
& +s^{-n \alpha} \bar{L}_{\alpha}\left[L_{\alpha}\left(v_{n}(x, t)\right)+N_{\alpha}\left(v_{n}(x, t)\right)\right. \\
& +f(x, t)]\} .
\end{aligned}
$$

This is the iteration formula for (6).

Example 4. To illustrate the above method, we can consider the following linear equation:

$$
\frac{d^{\alpha} v(x, t)}{d x^{\alpha}}=a v(x, t),
$$

$$
0<x \leq T, 0<\alpha \leq 1, a \in R .
$$

We construct the following iteration formula with the help of (12):

$$
v_{n+1}(x, t)=v_{n}(0, t)-\bar{L}_{\alpha}^{-1}\left[s^{-\alpha} \bar{L}_{\alpha}\left\{a v_{n}(x, t)\right\}\right] .
$$

Now, applying local fractional Laplace transform to the above equation, find

$$
v_{n+1}(x, t)=v_{n}(0, t)-\bar{L}_{\alpha}^{-1}\left[s^{-2 \alpha}\left\{a v_{n}(s, t)\right\}\right] .
$$

This is the iteration formula for (13).

Let us start from $v_{0}(x, t)=v(0, t)=v_{0}$.

Now, by putting the values of " $n$," we get the iterations; for $v_{1}(x, t)$, put $n=0$ in (15), and solving inverse local fractional Laplace transform, we have

$$
v_{1}(x, t)=v_{0}-\frac{a x^{\alpha} v_{0}}{\Gamma(1+\alpha)} .
$$


For $n=1, v_{2}(x)$ is given by the following iteration:

$$
v_{2}(x, t)=v_{1}(0, t)-\bar{L}_{\alpha}^{-1}\left[s^{-2 \alpha}\left\{a v_{1}(s, t)\right\}\right] .
$$

Using (16) and after easy calculations, we get

$$
v_{2}(x, t)=v_{0}-\frac{a x^{\alpha} v_{0}}{\Gamma(1+\alpha)}+\frac{\left(a x^{\alpha}\right)^{2} v_{0}}{\Gamma(1+2 \alpha)} .
$$

Similarly for $n=2, v_{2}(x)$ is given by the following iteration:

$$
\begin{gathered}
v_{3}(x)=v_{0}-\frac{a x^{\alpha} v_{0}}{\Gamma(1+\alpha)}+\frac{\left(a x^{\alpha}\right)^{2} v_{0}}{\Gamma(1+2 \alpha)}-\frac{\left(a x^{\alpha}\right)^{3} v_{0}}{\Gamma(1+3 \alpha)} \\
\vdots \\
v(x)=\sum_{n=0}^{\infty} v_{n}(x)=v_{0} \sum_{k=0}^{\infty} \frac{(-a)^{k} x^{k \alpha}}{\Gamma(1+k \alpha)} .
\end{gathered}
$$

This is the exact solution of (13).

Example 5. The following space-time fractional homogeneous telegraph equation can also be solved by the above introduced local fractional Laplace variational iteration method:

$$
\begin{array}{r}
\frac{\partial^{2 \alpha} v}{\partial x^{2 \alpha}} v(x, t)=\frac{\partial^{2 \alpha} v}{\partial t^{2 \alpha}}(x, t)+\frac{\partial^{\alpha} v}{\partial t^{\alpha}}(x, t)-v(x, t), \\
x, t \geq 0,0<\alpha \leq 1
\end{array}
$$

with initial conditions

$$
\begin{gathered}
v(0, t)=E_{\alpha}\left(-t^{\alpha}\right), \\
v^{\alpha}(0, t)=E_{\alpha}\left(-t^{\alpha}\right) .
\end{gathered}
$$

We can find the iteration formula for the above with the help of (12) as

$$
\begin{aligned}
& v_{n+1}(x, t)=\bar{L}_{\alpha}^{-1}\left[\sum_{k=1}^{n} s^{(k-1-n) \alpha} v_{n}^{(n-k) \alpha}(0, t)\right] \\
& +\bar{L}_{\alpha}^{-1}\left[s ^ { - 2 \alpha } \left\{\overline { L } _ { \alpha } \left[\frac{\partial^{2 \alpha} v_{n}}{\partial t^{2 \alpha}}(x, t)+\frac{\partial^{\alpha} v_{n}}{\partial t^{\alpha}}(x, t)\right.\right.\right. \\
& \left.\left.\left.-v_{n}(x, t)\right]\right\}\right] .
\end{aligned}
$$

Consider initial iteration as follows:

$$
\begin{gathered}
v_{0}(x, t)=\bar{L}_{\alpha}^{1}\left[\sum_{k=1}^{n} s^{(k-1-n) \alpha} v^{(n-k) \alpha}(0, t)\right], \\
\bar{L}_{\alpha}^{1}\left[s^{-\alpha} v(0, t)+s^{-2 \alpha} v^{\alpha}(0, t)\right] \\
=E_{\alpha}\left(-t^{\alpha}\right)+\frac{x^{\alpha}}{\Gamma(1+\alpha)} E_{\alpha}\left(-t^{\alpha}\right) .
\end{gathered}
$$

Now, by putting the values of " $n$," we get the iterations; for $v_{1}(x, t)$, put $n=0$ in (22); we have

$$
\begin{aligned}
& v_{1}(x, t)=\bar{L}_{\alpha}^{-1}\left[\sum_{k=1}^{n} s^{(k-1-n) \alpha} v_{0}^{(n-k) \alpha}(0, t)\right] \\
& +\bar{L}_{\alpha}^{-1}\left[s ^ { - 2 \alpha } \left\{\overline { L } _ { \alpha } \left[\frac{\partial^{2 \alpha} v_{0}}{\partial t^{2 \alpha}}(x, t)+\frac{\partial^{\alpha} v_{0}}{\partial t^{\alpha}}(x, t)\right.\right.\right. \\
& \left.\left.\left.\quad-v_{0}(x, t)\right]\right\}\right] .
\end{aligned}
$$

Using (23) and applying local fractional Laplace and inverse local fractional Laplace transform, we get

$$
\begin{aligned}
v_{1}(x, t)= & E_{\alpha}\left(-t^{\alpha}\right)+\frac{x^{\alpha}}{\Gamma(1+\alpha)} E_{\alpha}\left(-t^{\alpha}\right) \\
& -\frac{x^{2 \alpha}}{\Gamma(1+2 \alpha)} E_{\alpha}\left(-t^{\alpha}\right) \\
& -\frac{x^{3 \alpha}}{\Gamma(1+3 \alpha)} E_{\alpha}\left(-t^{\alpha}\right) .
\end{aligned}
$$

Similarly, we can find

$$
\begin{aligned}
& v_{2}(x, t)=E_{\alpha}\left(-t^{\alpha}\right)+\frac{x^{\alpha}}{\Gamma(1+\alpha)} E_{\alpha}\left(-t^{\alpha}\right) \\
& -\frac{x^{2 \alpha}}{\Gamma(1+2 \alpha)} E_{\alpha}\left(-t^{\alpha}\right) \\
& -\frac{x^{3 \alpha}}{\Gamma(1+3 \alpha)} E_{\alpha}\left(-t^{\alpha}\right) \\
& +\frac{x^{4 \alpha}}{\Gamma(1+4 \alpha)} E_{\alpha}\left(-t^{\alpha}\right) \\
& +\frac{x^{5 \alpha}}{\Gamma(1+5 \alpha)} E_{\alpha}\left(-t^{\alpha}\right) \text {, } \\
& v_{3}(x, t)=E_{\alpha}\left(-t^{\alpha}\right)+\frac{x^{\alpha}}{\Gamma(1+\alpha)} E_{\alpha}\left(-t^{\alpha}\right) \\
& -\frac{x^{2 \alpha}}{\Gamma(1+2 \alpha)} E_{\alpha}\left(-t^{\alpha}\right) \\
& -\frac{x^{3 \alpha}}{\Gamma(1+3 \alpha)} E_{\alpha}\left(-t^{\alpha}\right) \\
& +\frac{x^{4 \alpha}}{\Gamma(1+4 \alpha)} E_{\alpha}\left(-t^{\alpha}\right) \\
& +\frac{x^{5 \alpha}}{\Gamma(1+5 \alpha)} E_{\alpha}\left(-t^{\alpha}\right) \\
& -\frac{x^{6 \alpha}}{\Gamma(1+6 \alpha)} E_{\alpha}\left(-t^{\alpha}\right) \\
& -\frac{x^{7 \alpha}}{\Gamma(1+7 \alpha)} E_{\alpha}\left(-t^{\alpha}\right) \text {. }
\end{aligned}
$$


Consequently, we obtain

$$
\begin{gathered}
v(x, t)=E_{\alpha}\left(-t^{\alpha}\right)\left[1-\frac{x^{2 \alpha}}{\Gamma(1+2 \alpha)}+\frac{x^{4 \alpha}}{\Gamma(1+4 \alpha)}\right. \\
\left.-\frac{x^{6 \alpha}}{\Gamma(1+6 \alpha)}+\cdots\right]+E_{\alpha}\left(-t^{\alpha}\right)\left[\frac{x^{\alpha}}{\Gamma(1+\alpha)}\right. \\
\left.-\frac{x^{3 \alpha}}{\Gamma(1+3 \alpha)}+\frac{x^{5 \alpha}}{\Gamma(1+5 \alpha)}-\frac{x^{7 \alpha}}{\Gamma(1+7 \alpha)}+\cdots\right], \\
v(x, t)=E_{\alpha}\left(-t^{\alpha}\right) \cos _{\alpha}\left(x^{\alpha}\right)+E_{\alpha}\left(-t^{\alpha}\right) \sin _{\alpha}\left(x^{\alpha}\right) .
\end{gathered}
$$

Remark 6. The result in (27) is the same as the result obtained by Jafari and Jassim [17].

Example 7. The following space-time fractional homogeneous telegraph equation can also be solved by the above introduced local fractional Laplace variational iteration method:

$$
\begin{aligned}
& \frac{\partial^{2 \alpha} v}{\partial x^{2 \alpha}} v(x, t)=\frac{\partial^{2 \alpha} v}{\partial t^{2 \alpha}}(x, t)+2 \frac{\partial^{\alpha} v}{\partial t^{\alpha}}(x, t)+v(x, t), \\
& x, t \geq 0,0<\alpha \leq 1
\end{aligned}
$$

with initial conditions

$$
\begin{aligned}
v(0, t) & =1-E_{\alpha}\left(-t^{\alpha}\right), \\
\frac{\partial^{\alpha}}{\partial x^{\alpha}} v(0, t) & =0 .
\end{aligned}
$$

Then, we can find the iteration formula for the above with the help of (12) as

$$
\begin{aligned}
& v_{n+1}(x, t)=\bar{L}_{\alpha}^{-1}\left[\sum_{k=1}^{n} s^{(k-1-n) \alpha} v_{n}^{(n-k) \alpha}(0, t)\right] \\
& +\bar{L}_{\alpha}^{-1}\left[s ^ { - 2 \alpha } \left\{\overline { L } _ { \alpha } \left[\frac{\partial^{2 \alpha} v_{n}}{\partial t^{2 \alpha}}(x, t)+2 \frac{\partial^{\alpha} v_{n}}{\partial t^{\alpha}}(x, t)\right.\right.\right. \\
& \left.\left.\left.+v_{n}(x, t)\right]\right\}\right] .
\end{aligned}
$$

Consider initial iteration as follows:

$$
\begin{aligned}
& v_{0}(x, t)=\bar{L}_{\alpha}^{-1}\left[\sum_{k=1}^{n} s^{(k-1-n) \alpha} v^{(n-k) \alpha}(0, t)\right], \\
& \bar{L}_{\alpha}^{-1}\left[s^{-\alpha} v(0, t)+s^{-2 \alpha} v^{\alpha}(0, t)\right]=1-E_{\alpha}\left(-t^{\alpha}\right) .
\end{aligned}
$$

Now, by putting the values of " $n$," we get the iterations; for $v_{1}(x, t)$, put $n=0$ in (30); we have

$$
\begin{aligned}
& v_{1}(x, t)=\bar{L}_{\alpha}^{-1}\left[\sum_{k=1}^{n} s^{(k-1-n) \alpha} v_{0}^{(n-k) \alpha}(0, t)\right] \\
& +\bar{L}_{\alpha}^{-1}\left[s ^ { - 2 \alpha } \left\{\overline { L } _ { \alpha } \left[\frac{\partial^{2 \alpha} v_{0}}{\partial t^{2 \alpha}}(x, t)+2 \frac{\partial^{\alpha} v_{0}}{\partial t^{\alpha}}(x, t)\right.\right.\right. \\
& \left.\left.\left.+v_{0}(x, t)\right]\right\}\right] .
\end{aligned}
$$

Using (31) and applying local fractional Laplace and inverse local fractional Laplace transform, we get

$$
v_{1}(x, t)=1-E_{\alpha}\left(-t^{\alpha}\right)+\frac{x^{2 \alpha}}{\Gamma(1+2 \alpha)} .
$$

Similarly, we can find

$$
\begin{aligned}
& v_{2}(x, t)=1-E_{\alpha}\left(-t^{\alpha}\right)+\frac{x^{2 \alpha}}{\Gamma(1+2 \alpha)}-\frac{x^{4 \alpha}}{\Gamma(1+4 \alpha)} \\
& v_{3}(x, t)=1-E_{\alpha}\left(-t^{\alpha}\right)+\frac{x^{2 \alpha}}{\Gamma(1+2 \alpha)}+\frac{x^{4 \alpha}}{\Gamma(1+4 \alpha)} \\
& +\frac{x^{6 \alpha}}{\Gamma(1+6 \alpha)}
\end{aligned}
$$

Consequently, we obtain

$$
\begin{aligned}
& v(x, t)=\left[1-E_{\alpha}\left(-t^{\alpha}\right)\right]\left[1+\frac{x^{2 \alpha}}{\Gamma(1+2 \alpha)}+\frac{x^{4 \alpha}}{\Gamma(1+4 \alpha)}\right. \\
& \left.+\frac{x^{6 \alpha}}{\Gamma(1+6 \alpha)}+\cdots\right] \\
& v(x, t)=\cosh _{\alpha}\left(x^{\alpha}\right)-E_{\alpha}\left(-t^{\alpha}\right) \text {. }
\end{aligned}
$$

Example 8. We consider the following local fractional Laplace equation:

$$
\frac{\partial^{2 \alpha} v}{\partial x^{2 \alpha}} v(x, t)+\frac{\partial^{2 \alpha} v}{\partial t^{2 \alpha}}(x, t)=0, \quad x, t \geq 0,0<\alpha \leq 1
$$

with the initial condition

$$
\begin{aligned}
v(0, t) & =-E_{\alpha}\left(t^{\alpha}\right), \\
\frac{\partial^{\alpha}}{\partial x^{\alpha}} v(0, t) & =0 .
\end{aligned}
$$

Then, we can find the iteration formula for the above with the help of (12) as

$$
\begin{aligned}
v_{n+1}(x, t)=\bar{L}_{\alpha}^{-1} & {\left[\sum_{k=1}^{n} s^{(k-1-n) \alpha} v_{n}^{(n-k) \alpha}(0, t)\right] } \\
& +\bar{L}_{\alpha}^{-1}\left[s^{-2 \alpha}\left\{\bar{L}_{\alpha}\left[-\frac{\partial^{2 \alpha} v_{n}}{\partial t^{2 \alpha}}(x, t)\right]\right\}\right] .
\end{aligned}
$$

Consider initial iteration as follows:

$$
\begin{aligned}
& v_{0}(x, t)=\bar{L}_{\alpha}^{-1}\left[\sum_{k=1}^{n} s^{(k-1-n) \alpha} v^{(n-k) \alpha}(0, t)\right], \\
& \bar{L}_{\alpha}^{-1}\left[s^{-\alpha} v(0, t)+s^{-2 \alpha} v^{\alpha}(0, t)\right]=-E_{\alpha}\left(t^{\alpha}\right) .
\end{aligned}
$$


Now, by putting the values of " $n$," we get the iterations; for $v_{1}(x, t)$, put $n=0$ in (38); we have

$$
\begin{aligned}
v_{1}(x, t)= & \bar{L}_{\alpha}^{-1}\left[\sum_{k=1}^{n} s^{(k-1-n) \alpha} v_{0}^{(n-k) \alpha}(0, t)\right] \\
& +\bar{L}_{\alpha}^{-1}\left[s^{-2 \alpha}\left\{\bar{L}_{\alpha}\left[-\frac{\partial^{2 \alpha} v_{0}}{\partial t^{2 \alpha}}(x, t)\right]\right\}\right] .
\end{aligned}
$$

Using (39) and applying local fractional Laplace and inverse local fractional Laplace transform, we get

$$
v_{1}(x, t)=-E_{\alpha}\left(t^{\alpha}\right)+\frac{x^{2 \alpha}}{\Gamma(1+2 \alpha)} E_{\alpha}\left(t^{\alpha}\right) .
$$

Similarly, we can find

$$
\begin{aligned}
& v_{2}(x, t)=-E_{\alpha}\left(t^{\alpha}\right)+\frac{x^{2 \alpha}}{\Gamma(1+2 \alpha)} E_{\alpha}\left(t^{\alpha}\right) \\
& -\frac{x^{4 \alpha}}{\Gamma(1+4 \alpha)} E_{\alpha}\left(t^{\alpha}\right), \\
& v_{3}(x, t)=-E_{\alpha}\left(t^{\alpha}\right)+\frac{x^{2 \alpha}}{\Gamma(1+2 \alpha)} E_{\alpha}\left(t^{\alpha}\right) \\
& -\frac{x^{4 \alpha}}{\Gamma(1+4 \alpha)} E_{\alpha}\left(t^{\alpha}\right)+\frac{x^{6 \alpha}}{\Gamma(1+6 \alpha)} E_{\alpha}\left(t^{\alpha}\right)
\end{aligned}
$$

Consequently, we obtain

$$
\begin{aligned}
& v(x, t)=\left[-E_{\alpha}\left(t^{\alpha}\right)\right]\left[1-\frac{x^{2 \alpha}}{\Gamma(1+2 \alpha)}+\frac{x^{4 \alpha}}{\Gamma(1+4 \alpha)}\right. \\
& \left.-\frac{x^{6 \alpha}}{\Gamma(1+6 \alpha)}+\cdots\right], \\
& v(x, t)=-E_{\alpha}\left(t^{\alpha}\right) \cos _{\alpha}\left(x^{\alpha}\right) .
\end{aligned}
$$

Example 9. We consider the following local fractional Laplace equation:

$$
\frac{\partial^{2 \alpha} v}{\partial x^{2 \alpha}} v(x, t)+\frac{\partial^{2 \alpha} v}{\partial t^{2 \alpha}}(x, t)=0
$$

with the initial condition

$$
\begin{aligned}
v(0, t) & =0, \\
\frac{\partial^{\alpha}}{\partial x^{\alpha}} v(0, t) & =-E_{\alpha}\left(t^{\alpha}\right) .
\end{aligned}
$$

Then, we can find the iteration formula for the above with the help of (12) as

$$
\begin{aligned}
v_{n+1}(x, t)= & \bar{L}_{\alpha}^{-1}\left[\sum_{k=1}^{n} s^{(k-1-n) \alpha} v_{n}^{(n-k) \alpha}(0, t)\right] \\
& +\bar{L}_{\alpha}^{-1}\left[s^{-2 \alpha}\left\{\bar{L}_{\alpha}\left[-\frac{\partial^{2 \alpha} v_{n}}{\partial t^{2 \alpha}}(x, t)\right]\right\}\right] .
\end{aligned}
$$

Consider initial iteration as follows:

$$
\begin{aligned}
& v_{0}(x, t)=\bar{L}_{\alpha}^{-1}\left[\sum_{k=1}^{n} s^{(k-1-n) \alpha} v^{(n-k) \alpha}(0, t)\right], \\
& \bar{L}_{\alpha}^{-1}\left[s^{-\alpha} v(0, t)+s^{-2 \alpha} v^{\alpha}(0, t)\right]=-\frac{x^{\alpha}}{\Gamma(1+\alpha)} E_{\alpha}\left(t^{\alpha}\right) .
\end{aligned}
$$

Now, by putting the values of " $n$," we get the iterations; for $v_{1}(x, t)$, put $n=0$ in (46); we have

$$
\begin{aligned}
v_{1}(x, t)= & \bar{L}_{\alpha}^{-1}\left[\sum_{k=1}^{n} s^{(k-1-n) \alpha} v_{0}^{(n-k) \alpha}(0, t)\right] \\
& +\bar{L}_{\alpha}^{-1}\left[s^{-2 \alpha}\left\{\bar{L}_{\alpha}\left[-\frac{\partial^{2 \alpha} v_{0}}{\partial t^{2 \alpha}}(x, t)\right]\right\}\right] .
\end{aligned}
$$

Using (47) and applying local fractional Laplace and inverse local fractional Laplace transform, we get

$$
v_{1}(x, t)=-\frac{x^{\alpha}}{\Gamma(1+\alpha)} E_{\alpha}\left(t^{\alpha}\right)+\frac{x^{3 \alpha}}{\Gamma(1+3 \alpha)} E_{\alpha}\left(t^{\alpha}\right) .
$$

Similarly, we can find

$$
\begin{aligned}
& v_{2}(x, t)=-\frac{x^{\alpha}}{\Gamma(1+\alpha)} E_{\alpha}\left(t^{\alpha}\right)+\frac{x^{3 \alpha}}{\Gamma(1+3 \alpha)} E_{\alpha}\left(t^{\alpha}\right) \\
& -\frac{x^{5 \alpha}}{\Gamma(1+5 \alpha)} E_{\alpha}\left(t^{\alpha}\right),
\end{aligned}
$$

$$
\begin{gathered}
v_{3}(x, t)=-\frac{x^{\alpha}}{\Gamma(1+\alpha)} E_{\alpha}\left(t^{\alpha}\right)+\frac{x^{3 \alpha}}{\Gamma(1+3 \alpha)} E_{\alpha}\left(t^{\alpha}\right) \\
-\frac{x^{5 \alpha}}{\Gamma(1+5 \alpha)} E_{\alpha}\left(t^{\alpha}\right)+\frac{x^{7 \alpha}}{\Gamma(1+7 \alpha)} E_{\alpha}\left(t^{\alpha}\right)
\end{gathered}
$$

Consequently, we obtain

$$
\begin{aligned}
& v(x, t)=\left[-E_{\alpha}\left(t^{\alpha}\right)\right] \\
& \cdot\left[\frac{x^{\alpha}}{\Gamma(1+2 \alpha)}-\frac{x^{3 \alpha}}{\Gamma(1+4 \alpha)}+\frac{x^{5 \alpha}}{\Gamma(1+6 \alpha)}-\cdots\right] \text {, } \\
& v(x, t)=-E_{\alpha}\left(t^{\alpha}\right) \sin _{\alpha}\left(x^{\alpha}\right) .
\end{aligned}
$$

\section{Conclusion}

In this work, we have presented successful demonstration of the local fractional Laplace variational iteration method for solutions of a wide class of problems. Analytical solutions of the telegraph and Laplace equations on Cantor sets involving local fractional derivatives are efficiently developed.

\section{Conflict of Interests}

The authors declare no conflict of interests. 


\section{Authors' Contribution}

Both authors have worked equally in this paper. Both authors have read and approved the final paper.

\section{References}

[1] A. A. Kilbas, H. M. Srivastava, and J. J. Trujillo, Theory and Applications of Fractional Differential Equations, Elsevier, Amsterdam, The Netherlands, 2006.

[2] K. S. Miller and B. Ross, An Introduction to the Fractional Calculus and Fractional Differential Equations, Wiley, New York, NY, USA, 1993.

[3] K. B. Oldham and J. Spanier, The Fractional Calculus, Academic Press, New York, NY, USA, 1974.

[4] V. Turut and N. Güzel, "On solving partial differential equations of fractional order by using the variational iteration method and multivariate padé approximations," European Journal of Pure and Applied Mathematics, vol. 6, no. 2, pp. 147-171, 2013.

[5] X. J. Yang, Advanced Local Fractional Calculus and Its Applications, World Science Publisher, New York, NY, USA, 2012.

[6] A.-M. Yang, J. Li, H. M. Srivastava, G.-N. Xie, and X.-J. Yang, "Local fractional Laplace variational iteration method for solving linear partial differential equations with local fractional derivative," Discrete Dynamics in Nature and Society, vol. 2014, Article ID 365981, 8 pages, 2014.

[7] X. J. Yang, "Local fractional integral transforms," Progress in Nonlinear Science, vol. 4, pp. 12-25, 2011.

[8] X. J. Yang, Local Fractional Functional Analysis and Its Applications, Asian Academic Publisher Limited, Hong Kong, China, 2011.

[9] F. B. M. Belgacem and A. A. Karaballi, "Sumudu transform fundamental properties investigations and applications," Journal of Applied Mathematics and Stochastic Analysis, vol. 2006, Article ID 91083, 23 pages, 2006.

[10] J. Chen, F. Liu, and V. Anh, "Analytical solution for the timefractional telegraph equation by the method of separating variables," Journal of Mathematical Analysis and Applications, vol. 338, no. 2, pp. 1364-1377, 2008.

[11] J. S. Duan, R. Rach, D. Baleanu, and A. M. Wazwaz, "A review of the Adomian decomposition method and its applications to fractional differential equations," Communications in Fractional Calculus, vol. 3, no. 2, pp. 73-99, 2012.

[12] M. Garg, P. Manohar, and S. L. Kalla, "Generalized differential transform method to space-time fractional telegraph equation," International Journal of Differential Equations, vol. 2011, Article ID 548982, 9 pages, 2011.

[13] J.-H. He, "Variational iteration method-a kind of non-linear analytical technique: some examples," International Journal of Non-Linear Mechanics, vol. 34, no. 4, pp. 699-708, 1999.

[14] Y. Khan, J. Diblík, N. Faraz, and Z. Šmarda, "An efficient new perturbative Laplace method for space-time fractional telegraph equations," Advances in Difference Equations, vol. 2012, article 204, 2012.

[15] H. M. Srivastava, A. K. Golmankhaneh, D. Baleanu, and X.-J. Yang, "Local fractional Sumudu transform with application to IVPs on cantor sets," Abstract and Applied Analysis, vol. 2014, Article ID 620529, 7 pages, 2014.

[16] M. Inokuti, H. Sekine, and T. Mura, "General use of the Lagrange multiplier in nonlinear mathematical physics," in Variational Methods in the Mechanics of Solids, S. Nemat-Nasser, Ed., pp. 156-162, Pregman Press, New York, NY, USA, 1978.
[17] H. Jafari and H. K. Jassim, "Numerical solutions of telegraph and laplace equations on cantor sets using local fractional Laplace decomposition method," International Journal of Advances in Applied Mathematics and Mechanics, vol. 2, no. 3, pp. 144-151, 2015. 


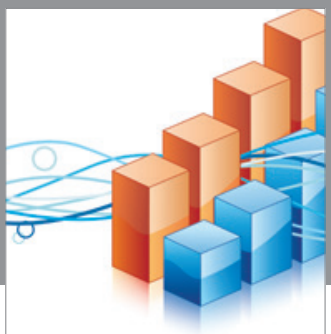

Advances in

Operations Research

vatem alat4

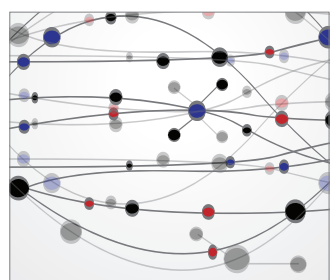

\section{The Scientific} World Journal
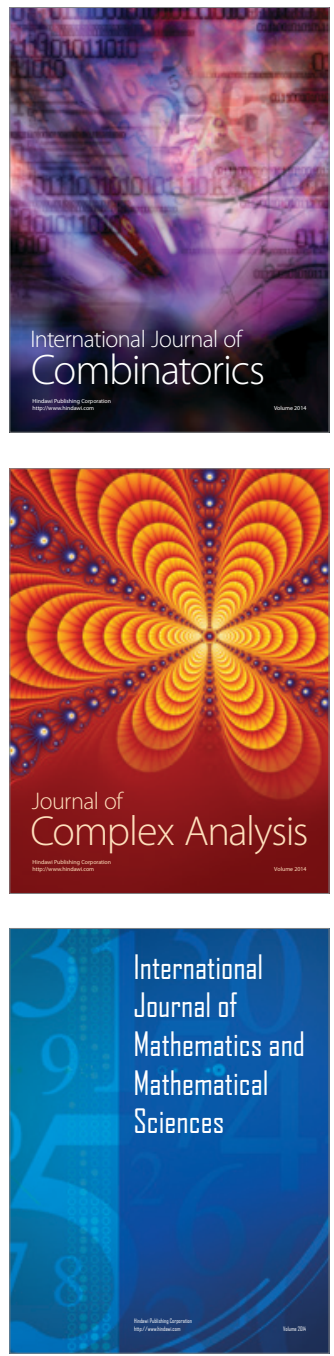
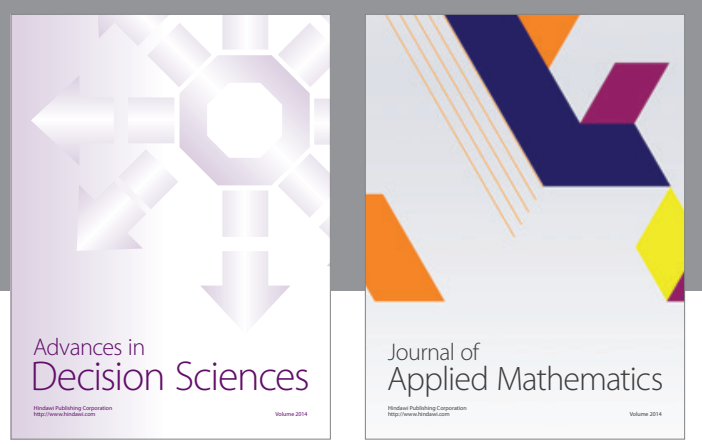

Algebra

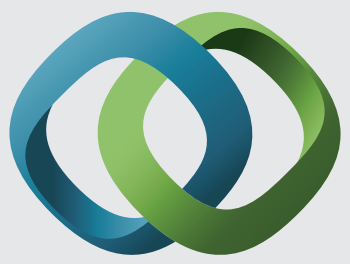

\section{Hindawi}

Submit your manuscripts at

http://www.hindawi.com
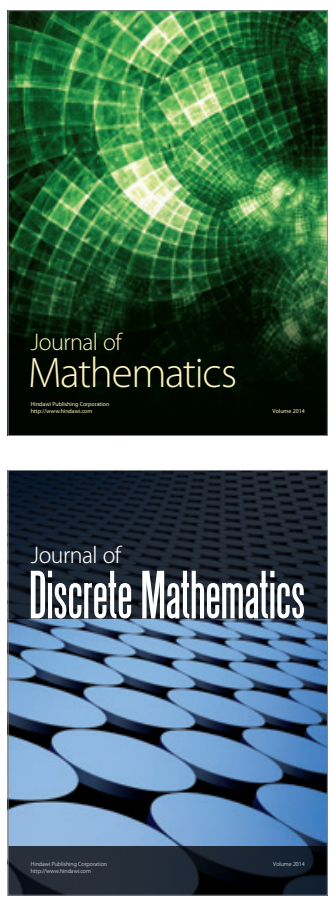

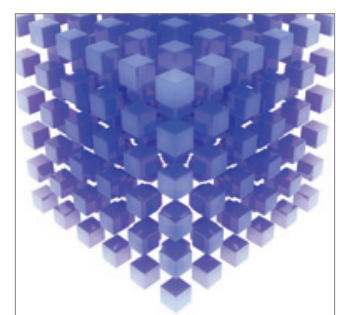

Mathematical Problems in Engineering
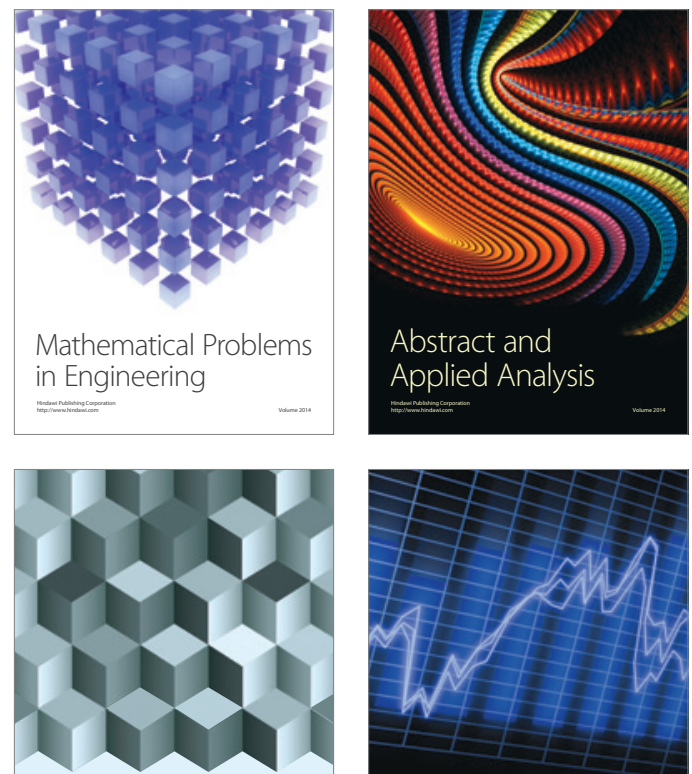

Journal of

Function Spaces

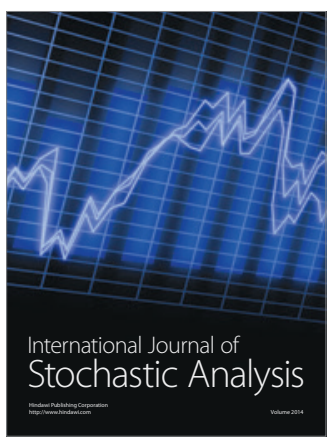

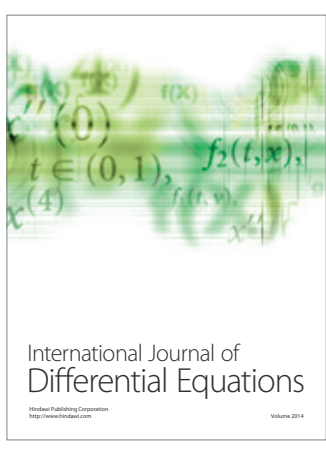
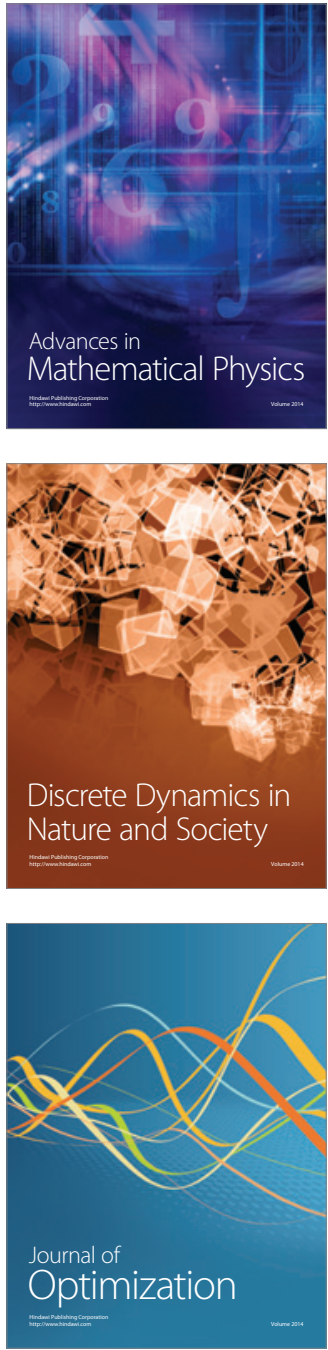